\title{
Novel functions of endocytic player clathrin in mitosis
}

\author{
Wenxiang $\mathrm{Fu}^{1}$, Qing Jiang ${ }^{1}$, Chuanmao Zhang ${ }^{1}$ \\ ${ }^{I}$ The MOE Key Laboratory of Cell Proliferation and Differentiation and the State Key Laboratory of Bio-membrane and Mem- \\ brane Biotechnology, College of Life Sciences, Peking University, Beijing 100871, China
}

\begin{abstract}
Clathrin has been widely recognized as a pivotal player in endocytosis, in which several adaptors and accessory proteins are involved. Recent studies suggested that clathrin is also essential for cell division. Here this review mainly focuses on the clathrin-dependent mechanisms involved in spindle assembly and chromosome alignment. In mitosis, clathrin forms a complex with phosphorylated TACC3 to ensure spindle stability and proper chromosome alignment. The clathrin-regulated mechanism in mitosis requires the crosstalk among clathrin, spindle assembly factors (SAFs), Ran-GTP and mitotic kinases. Meanwhile, a coordinated mechanism is required for role transitions of clathrin during endocytosis and mitosis. Taken together, the findings of the multiple functions of clathrin besides endocytosis have expanded our understanding of the basic cellular activities.
\end{abstract}

Keywords: clathrin; endocytosis; mitosis; TACC3; spindle assembly; chromosome alignment Cell Research (2011) 21:1655-1661. doi:10.1038/cr.2011.106; published online 28 June 2011

Clathrin-mediated endocytosis is a vesicular transport process for a variety of cellular events including signal transduction, nutrient import and synaptic vesicles [1]. To uptake materials from environment by this process, the first step for a cell is to organize the clathrin latticecoated vesicles $[2,3]$. Clathrin lattice is assembled from triskelions, each of which is constituted from three heavy chains (CHCs) and three light chains (CLCs) [4]. Once the site for the formation of the clathrin-coated vesicles is decided, the membrane initially curves and internalizes into small vesicle pits, and around the pits, clathrin and its various binding proteins organize the clathrin coats to form the vesicles. Then, scission of the clathrin-coated vesicles from the cell membrane enables cargos to be transported to the target place $[5,6]$.

Mitosis is the process of cell division to maintain genomic information in eukaryotes. During mitosis, the macromolecular machine known as the spindle segregates chromosomes to two daughter cells [7, 8]. In most eukaryotic cells, the mitotic spindle is composed of microtubules, centrosomes and chromosomes. The highly dynamic microtubules form a bipolar structure regulated

Correspondence: Chuanmao Zhang

Tel: 86-10-62757173; Fax: 86-10-62767246

E-mail: zhangcm@pku.edu.cn by a variety of motor proteins, the centrosomes function as the main microtubule sites in cells and chromosomes function in microtubule self-assembly and microtubule attachment [7]. Assembly of the mitotic spindle is orchestrated by the microtubule structure and the membranous spindle matrix together [9].

Clathrin was reported to be localized on mitotic spindles and function in mitosis a few years ago [10-14]. More recently, the role of clathrin in mitosis has been widely recognized. Several individual groups including ours have shed novel lights on the function of clathrin in mitosis at the molecular level [15-18]. These studies demonstrated that clathrin forms a complex with TACC3 to regulate spindle assembly and chromosome alignment. In this paper, we mainly discuss the progress on the regulation mechanisms of clathrin in mitotic spindle assembly and chromosome alignment and the relations between clathrin-dependent endocytosis and mitosis.

\section{Clathrin and mitotic spindle assembly}

The assembly of spindle apparatus in mitosis guarantees one cell to produce two genetically identical daughter cells. During the process of spindle assembly, the endocytic protein clathrin has been supposed to be involved [12]. It was found that clathrin is localized on spindle during cell division [10-14]. Clathrin knockdown by RNAi causes defects in spindle assembly, suggesting that 
clathrin may play a role in spindle assembly. B-Myb is a transcription factor in vertebrate cells and its knockdown by RNAi abolishes the localization of clathrin on mitotic spindles [14]. It was proposed that the B-Myb complex containing clathrin and filamin together contributes to the function of mitotic spindle and normal mitotic progression [14]. Cyclin G-associated kinase (GAK), which was previously known to regulate clathrin in endocytosis, was also found to be required for the localization of clathrin on spindles $[19,20]$.

Recently, several groups found that clathrin regulates spindle assembly by targeting TACC 3 to the spindles (Figure 1A) [15-17]. During mitosis, Aurora A phosphorylates TACC 3 and this phosphorylation ensures TACC 3 to localize to the spindles in Drosophila, Xenopus and human [21-25]. The road leading to discovery of the novel function of clathrin in mitosis started from exploring how phosphorylation- catalyzed TACC 3 affects mitotic spindle assembly. By mass spectrometry analysis, clathrin was initially identified as one of the specific binding partners of phosphorylated TACC3 [15-17]. Through site-directed mutagenesis studies joining with biochemical and cell biological assays, it was demonstrated that the binding of $\mathrm{CHC}$ and phosphorylated TACC 3 is required for proper mitotic spindle assembly. In early mitosis, TACC 3 is activated through the phosphorylation by Aurora A. Soon after the activation, the phosphorylated TACC 3 forms a complex with $\mathrm{CHC}$ and together loads to spindles [15-17]. Subsequently, the CHC-TACC3 complex further recruits other molecules such as XMAP215/ ch-TOG to ensure spindle stability $[16,26]$.

Aurora A phosphorylates TACC3 at three sites (S33/ S620/S626 in Xenopus and S34/S552/S558 in humans) in vitro and phosphorylation of TACC 3 enables TACC 3 to target to spindle and poles for spindle assembly [23, $25,27]$. However, it was later found that only the phosphorylation of TACC 3 at the latter two sites are required for the formation of the clathrin-TACC 3 complex, its localization on spindle and poles and its function in mitotic spindle assembly both in vitro and in vivo [17]. It was reported that the CHC 331-542aa region, which is comprised of a linker region and the first clathrin heavy chain repeat CHCR0, directly binds with phosphorylated TACC3 to form clathrin-TACC3 complex [16]. In addition, CHC 1-479aa, especially 1-330aa, is essential for $\mathrm{CHC}$ to localize to spindles $[12,28]$. However, as trimerization of clathrin was proposed to be required for mitotic spindle assembly [28], whether and how the TACC3-bound $\mathrm{CHC}$ form a triskelion requires further study. As CLC was also detected by QUBIC method in TACC3 co-precipitates [15], it may be possible that the phosphorylated TACC 3 forms complexes with $\mathrm{CHC}$ attached by CLC, or even a clathrin triskelion. It also remains unknown whether the mitotic clathrin forms a lattice in binding with TACC3. Taken together, the functions of clathrin in mitosis to ensure spindle stability are achieved by targeting TACC 3 , which undergoes priming phosphorylation by Aurora A kinase, to spindle microtubules and spindle poles.

However, another recent paper by Royle and colleagues reported that, although the ablation of clathrin reduced TACC 3 targeting to the spindle, the depletion of TACC 3 also dramatically resulted in the reduced spindle localization of clathrin [18]. Similar to the previous report [17], they also observed that overexpression of TACC 3 results in the accumulation of clathrin at the spindle [18]. Therefore, the authors proposed a distinct hypothesis that TACC 3 recruits clathrin to the mitotic spindle microtubules [18]. They proposed that TACC3 and ch-TOG bind to the spindle microtubules under the regulation of Aurora A followed by the recruitment of clathrin to the microtubules through forming complex with TACC3 or TACC3/ch-TOG subcomplex. Then, the clathrin molecule in the complex may bind more than one TACC 3 or TACC $3 /$ ch-TOG subcomplex between adjacent parallel microtubules to form an inter-microtubule bridge that stabilize K-fibers of the spindle by physical crosslinking the microtubules and reducing their catastrophe rates (Figure 1B) [18]. However, the previous three papers reported that knockdown of TACC3 did not reduce the spindle localization of $\mathrm{CHC}$ [15-17]. Treatment with Aurora kinase inhibitor also did not result in the reduction of clathrin localization on the spindle although the spindle TACC3 was abolished [15]. Moreover, the facts that the $\mathrm{CHC}$ fragment 331-1639aa, which contains the TACC 3 interaction domain, failed to localize to the spindle $[16,28]$ and that CHC 1-330aa, which lacks the TACC3 interaction domain, was able to target to the spindle $[12,16]$ do not support the hypothesis that TACC3 recruits clathrin to the spindle. Therefore, the order of the recruitment of clathrin and TACC 3 to the mitotic spindle remains controversial and needs to be further clarified. Given that the conservation of the protein sequences of clathrin, Aurora A and TACC 3 in vertebrates, it is also very likely conserved that clathrin and TACC 3 form a complex to carry out their roles in mitosis.

\section{Clathrin regulates mitotic chromosome alignment}

Depletion of TACC3 by RNAi leads to chromosome misalignment and reduced localization of kinetochore proteins in mitosis, suggesting that TACC 3 is required for microtubule-kinetochore interaction [26, 29]. Meanwhile, clathrin was also found to be required for chromo- 


\section{A 'Clathrin recruits TACC3' model}
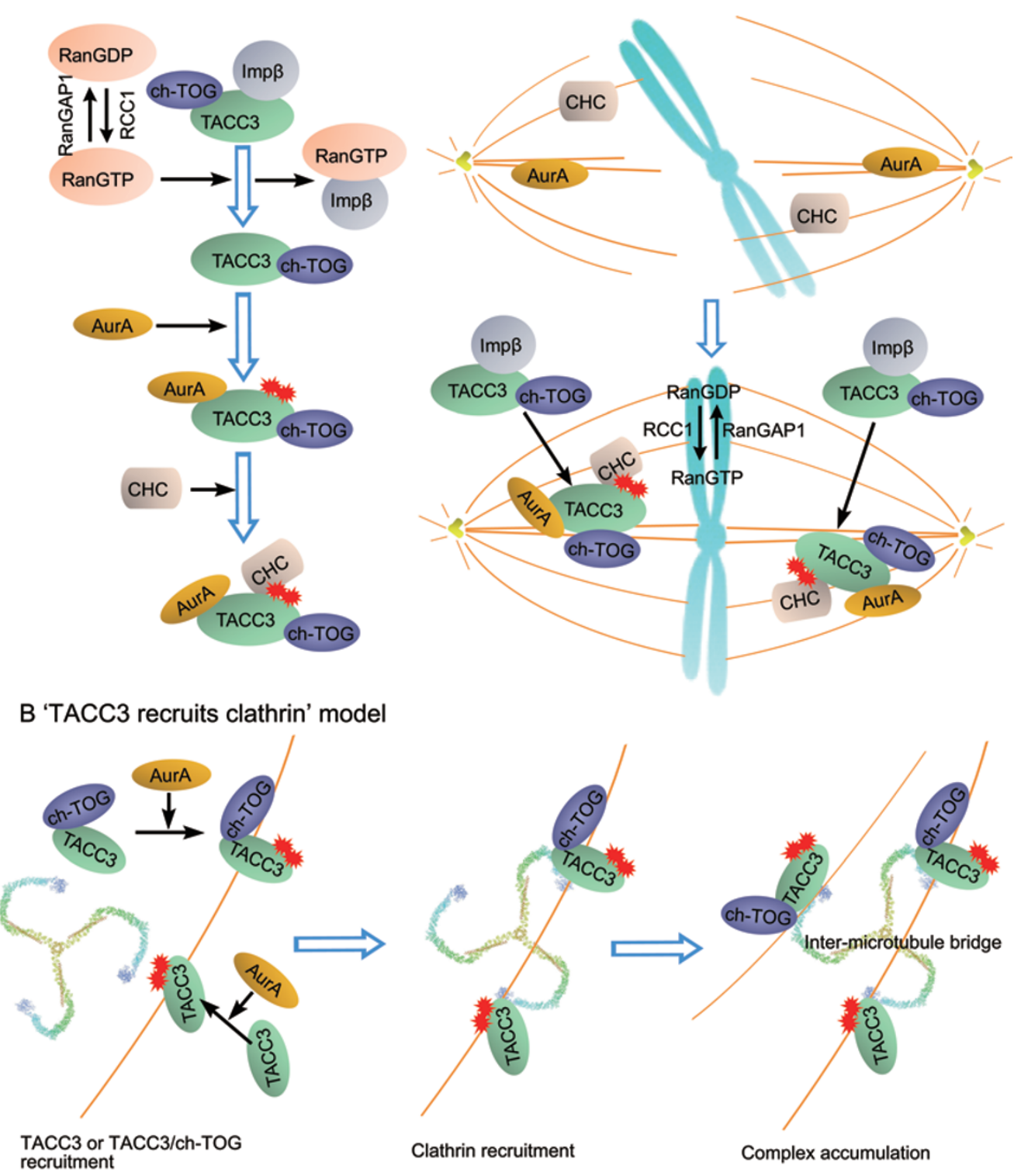

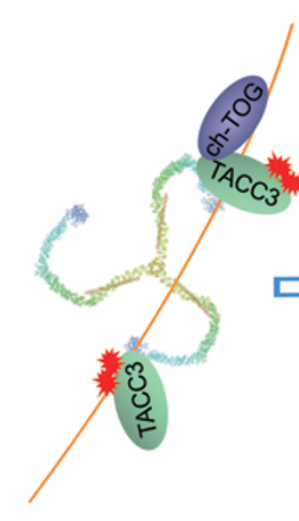

Clathrin recruitment

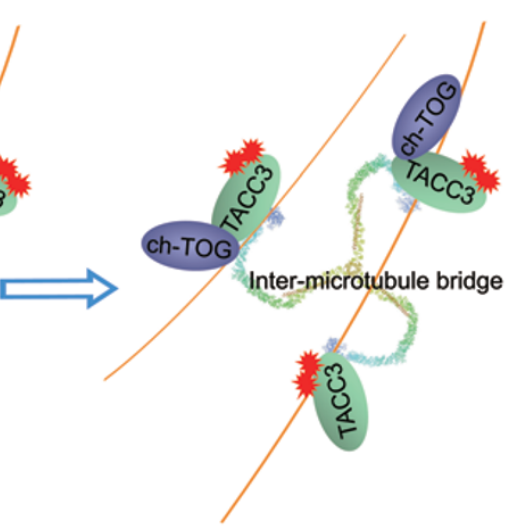

Complex accumulation

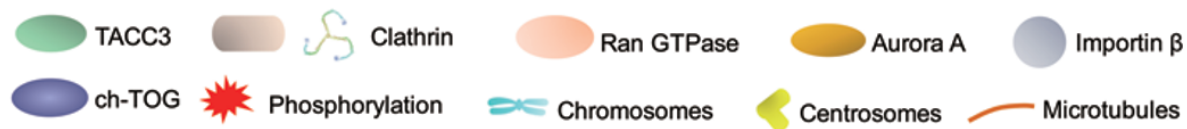

Figure 1 Clathrin-TACC3 complex regulates mitotic spindle assembly. Two models describing the function of clathrin-TACC3 complex in mitotic spindle assembly. (A) The 'clathrin recruits TACC3' model. The conversion between GTP-bound and GDPbound states of Ran GTPase is ensured by guanine nucleotide exchange factor RCC1 and Ran GTPase activating protein RanGAP1. When the cell enters mitosis, RanGTP is generated by RCC1 around the chromosomes. Through specific binding of RanGTP with importin- $\beta$, TACC3, one of the spindle assembly factors (SAFs), is released from the inhibitory binding with importin $\beta$. Then, Aurora A kinase activates the released TACC3 by phosphorylating it at two sites (Ser620 and Ser626 in Xenopus) and enables it to bind with clathrin to form the clathrin-TACC3 complex. Finally, the clathrin-associated TACC3 is targeted to spindle poles and spindle microtubules for proper spindle assembly. Along with TACC3, ch-TOG also targets to spindles to regulate spindle stability. (B) The 'TACC3 recruits clathrin model'. First, TACC3 alone or complexed with chTOG is recruited to the spindle microtubules in a phosphorylation-dependent manner regulated by Aurora A. Then, clathrin is recruited to the spindle by the spindle microtubule-localized TACC3 or TACC3/ch-TOG. Finally, a clathrin molecule forms an inter-microtubule bridge by interacting with additional TACC3 or TACC3/ch-TOG on adjacent microtubules to stabilize the spindle microtubules. 
some alignment. Knockdown of CHC by RNAi resulted in kinetochore fiber destabilization and defective congression of chromosomes to the metaphase plate [12]. More recently, it was found that TACC3 regulates chromosome alignment dependent on its phosphorylation by Aurora A and that $\mathrm{CHC}$ specifically recruits phosphorylated TACC 3 to the mitotic spindle and poles, linking the roles of clathrin and TACC 3 together in proper mitotic spindle assembly and chromosome alignment [15-17]. Although the order of the recruitment of clathrin and TACC3 remains controversial [18], it seems possible that clathrin and TACC 3 function together in mitosis. Moreover, the chromosome alignment defects caused by RNAi knockdown of TACC 3 can be rescued by expressing exogenous phospho-mimic TACC3, but not its nonphosphorylatable mutants. Considering that $\mathrm{CHC}$ targets phosphorylated TACC 3 to the mitotic spindle, it may be suggested that $\mathrm{CHC}$ regulates mitotic chromosome alignment also through forming clathrin-TACC 3 complex.

Knockdown of TACC3 by RNAi leads to reduced localization of structural and checkpoint proteins at kinetochores, mitotic index increase, prolonged mitosis and apoptosis [30, 31]. Knockdown of clathrin by RNAi leads to persistent activation of the spindle checkpoint as well as destabilization of kinetochore fibers [12]. The traditional view is that the defects of kinetochoremicrotubule attachment and tension can lead to activation of the spindle checkpoint $[30,31]$. However, as the CLC A interacts with the mitotic arrest deficient protein MAD2B during mitosis [32], the previous research could not rule out the possibility that clathrin-TACC 3 complex can directly regulate spindle checkpoint. Therefore, the clathrin-TACC3 complex probably regulates chromosome alignment by ensuring both proper kinetochoremicrotubule attachment and spindle assembly checkpoint [15-17]. Moreover, a complex of GAK and B-Myb, which functions in spindle assembly upstream of clathrin, is also involved in chromosome congression $[14,19$, 20]. However, it is not clear whether the failure of chromosome alignment in clathrin or TACC 3 knockdown studies is caused by the defects of the spindle assembly. Of course, the mechanisms may be much more complicated. Thus, understanding the role of clathrin could be pivotal to elucidate the real relationships between spindle assembly and chromosome alignment.

\section{Crosstalk among clathrin, SAFs, Ran and mitotic kinases in mitosis}

The spindle assembly is a very complicated process, which requires coordination of a variety of spindle assembly factors (SAFs), mitotic kinases, Ran GTPase and Ran-binding proteins [33]. The Ran GTPase cycle is regulated by RanGAP, the GTPase activity stimulator, and RCC1, the GDP-GTP exchange factor for Ran. Ran, with its regulators and effectors, was first recognized to regulate the nuclear transport between the nuclear envelope. The Ran system was then found to regulate the mitotic spindle assembly and the nuclear envelope assembly. RanGTP binds to nuclear transport factors and controls their association with cargos. When the cell enters mitosis from interphase, RanGTP regulates nuclear pore complex disassembly and nuclear envelope breakdown. The SAFs, which usually contain nuclear localization sequences, are targeted into nucleus in interphase by importin- $\alpha / \beta$ dimer, two Ran system factors that were firstly recognized as nuclear import regulators. In mitosis, the binding of SAFs with importin proteins would be inhibitory and the inhibition must be lifted for proper mitotic spindle assembly. Ran in its GTP-bound form could specifically bind with importin- $\beta$ and release the SAFs. So far a number of SAFs, such as TPX2, NuMA, Rae1 and TACC 3 , have been identified to be released from importins by RanGTP for mitotic spindle assembly [33].

Clathrin was previously reported to be required for the function of mitotic spindle and proposed to act as a brace between two or three microtubules within a kinetochore fiber to increase fiber stability $[12,28]$. The recent finding that clathrin specifically binds and recruits phosphorylated TACC 3 to spindle and poles for proper spindle assembly and chromosome alignment is an important progress in the field [15-17]. It may suggest a general mechanism for spindle assembly as well as chromosome alignment by SAFs, Ran and mitotic kinases (Figure 1). When the cell enters mitosis from G2, the inhibitory binding of SAFs with importins, here at least TACC3, will be released by RanGTP and immediately modified by mitotic kinases. The SAFs will then be recruited to the mitotic spindle and poles by additional regulator(s), here the clathrin protein triskelion. Once recruited, the SAFs alone or complexed with other factors will locally perform their mitotic functions. Therefore, the coordinated regulation among SAFs, clathrin, Ran and mitotic kinases will be a general mechanism for the mitotic spindle assembly and the proper chromosome alignment (Figure 1).

As reviewed, the interplay among clathrin, Aurora A and TACC 3 presents a crosstalk between clathrin and Aurora A kinase. Moreover, other studies also suggested the potential cooperation between mitotic kinases and clathrin. The endocytic protein Numb, which directly associates with clathrin-associated adaptor complex AP-2 [34], is phosphorylated by AAK1 to promote clathrincoated pits maturation [35]; while in asymmetric cell 
division, Numb is regulated by Aurora and Polo kinase: Aurora A phosphorylates Par6 for the release of Numb from posterior cell cortex and Polo phosphorylates Pon for the localization of Numb at the anterior cell cortex [36-38]. With no doubt, in the cell, the crosstalk among clathrin, Ran-GTP and mitotic kinases could be much more complicated than expected and more regulators need to be found.

\section{Role transitions of clathrin between endocytosis and mitosis}

The functions of clathrin in interphase and mitosis have simply taken endocytosis and mitosis into the same pool; however, the relationships and regulations between them must be complicated. A lot of previous work suggested that clathrin-mediated endocytosis is arrested during mitosis as other endocytic steps [39, 40]. Moreover, inhibition of clathrin-mediated endocytosis does not disrupt mitosis, suggesting that endocytosis and spindle assembly are two distinct processes [12]. However, it was found recently that clathrin-dependent internalization of materials on the cell plasma membrane takes place throughout the cell cycle, while the endosomal recycling is ceased in mitosis and enhanced in cytokinesis [41, 42]. Moreover, several other clathrin related membrane vesicle proteins were found recently to function in mitosis and in endocytosis. The clathrin adaptor protein AP2 was found to interact with the mitotic checkpoint kinase BubR1 [43]. Another endocytic clathrin-associated adaptor protein autosomal recessive hypercholesterolemia (ARH) functions to sort members of the LDL receptor superfamily (LDLR, megalin, LRP) and participates in centrosomal and mitotic dynamics by interacting with centrosomal proteins. Cyclin G-associated kinase (GAK) is known to be essential for clathrin-mediated membrane trafficking; while in mitosis, cells lacking GAK show strongly reduced levels of clathrin on the mitotic spindle. GAK and clathrin function cooperatively in centrosome maturation, chromosome congression, spindle assembly and microtubule outgrowth from kinetochores/chromosomes $[19,20]$. Moreover, the major function of GAK at spindles is probably performed by recycling clathrin from endocytic vesicles at the onset of mitosis [20]. Taken together, these findings suggest that some endocytic players including clathrin switch to function in spindle assembly when the cell enters mitosis.

Recently, the functions of epsin in mitosis may provide a new insight into the role transition between spindle assembly and endocytosis. Epsin directly modifies membrane curvature in binding to the membrane lipid PtdIns(4,5)P2 in conjunction with clathrin polym- erization and limits clathrin coat assembly to the size of newly formed vesicles $[44,45]$. The membrane deformation function of epsin contributes to spindle organization during mitosis [46].As spindle matrix proteomics has revealed several endocytosis proteins [47, 48], we believe that endocytic players, which were previously thought to be outside the spindles, may also switch to function in microtubule organization inside the spindles in a very complicated way [46]. Furthermore, quantitative phosphorproteomics suggests that some endocytic partners including clathrin are regulated by phosphorylation throughout the cell cycle $[49,50]$, implicating that both kinases and phosphatases may coordinate the switch between endocytosis and mitosis through regulating phosphorylation state of endocytic regulators.

\section{Conclusions and prospects}

Besides playing a pivotal role in endocytosis, clathrin also regulates spindle assembly and chromosome alignment. However, there is still a long way to go to fully understand the roles of clathrin during the cell cycle at the molecular, structural and evolutionary levels. Except for the well documented roles of clathrin in the coated membrane vesicle formation in endocytosis, how clathrin play roles in other processes in the cell remains mysterious. Moreover, when the cell is in the different stages of the cell cycle, how the functions of clathrin are coordinated is also a big question. The last but not the least, the function of clathrin in tumorigenesis needs to be further elucidated [51].

\section{Acknowledgments}

We thank all the other members of our laboratory for valuable comments. This work was supported by grants from the National Natural Science Foundation of China (30900726, 31071188, 30721064, 31030044 and 90913021) and the State Key Basic Research and Development Plan (2006CB910101, 2007CB914502 and 2010CB833705).

\section{References}

1 Takei K, Haucke V. Clathrin-mediated endocytosis: membrane factors pull the trigger. Trends Cell Biol 2001; 11:385-391.

2 Edeling MA, Smith C, Owen D. Life of a clathrin coat: insights from clathrin and AP structures. Nat Rev Mol Cell Biol 2006; 7:32-44.

3 Ungewickell EJ, Hinrichsen L. Endocytosis: clathrin-mediated membrane budding. Curr Opin Cell Biol 2007; 19:417-425.

4 Fotin A, Cheng Y, Sliz P, et al. Molecular model for a complete clathrin lattice from electron cryomicroscopy. Nature 2004; 432:573-579.

5 Schmid EM, McMahon HT. Integrating molecular and net- 
work biology to decode endocytosis. Nature 2007; 448:883888.

6 Rappoport JZ. Focusing on clathrin-mediated endocytosis. Biochem J 2008; 412:415-423.

7 Walczak CE, Heald R. Mechanisms of mitotic spindle assembly and function. Int Rev Cytol 2008; 265:111-158.

8 Wittmann T, Hyman A, Desai A. The spindle: a dynamic assembly of microtubules and motors. Nat Cell Biol 2001; 3:E28-E34.

9 Zheng Y. A membranous spindle matrix orchestrates cell division. Nat Rev Mol Cell Biol 2010; 11:529-535.

10 Maro B, Johnson MH, Pickering SJ, Louvard D. Changes in the distribution of membranous organelles during mouse early development. J Embryol Exp Morphol 1985; 90:287-309.

11 Okamoto CT, McKinney J, Jeng YY. Clathrin in mitotic spindles. Am J Physiol Cell Physiol 2000; 279:C369-C374.

12 Royle SJ, Bright NA, Lagnado L. Clathrin is required for the function of the mitotic spindle. Nature 2005; 434:1152-1157.

13 Tahara H, Yokota E, Igarashi $\mathrm{H}$, et al. Clathrin is involved in organization of mitotic spindle and phragmoplast as well as in endocytosis in tobacco cell cultures. Protoplasma 2007; 230:111.

14 Yamauchi T, Ishidao T, Nomura T, et al. A B-Myb complex containing clathrin and filamin is required for mitotic spindle function. $E M B O$ J 2008; 27:1852-1862.

15 Hubner NC, Bird AW, Cox J, et al. Quantitative proteomics combined with BAC TransgeneOmics reveals in vivo protein interactions. J Cell Biol 2010; 189:739-754.

16 Lin $\mathrm{CH}, \mathrm{Hu} \mathrm{CK}$, Shih HM. Clathrin heavy chain mediates TACC 3 targeting to mitotic spindles to ensure spindle stability. J Cell Biol 2010; 189:1097-1105.

$17 \mathrm{Fu} \mathrm{W,} \mathrm{Tao} \mathrm{W,} \mathrm{Zheng} \mathrm{P,} \mathrm{et} \mathrm{al.} \mathrm{Clathrin} \mathrm{recruits} \mathrm{phosphorylated}$ TACC3 to spindle poles for bipolar spindle assembly and chromosome alignment. J Cell Sci 2010; 123:3645-3651.

18 Booth DG, Hood FE, Prior IA, Royle SJ. A TACC3/ch-TOG/ clathrin complex stabilises kinetochore fibres by inter-microtubule bridging. EMBO J 2011; 30:906-919

19 Shimizu H, Nagamori I, Yabuta N, Nojima H. GAK, a regulator of clathrin-mediated membrane traffic, also controls centrosome integrity and chromosome congression. J Cell Sci 2009; 122:3145-3152.

20 Tanenbaum ME, Vallenius T, Geers EF, et al. Cyclin G-associated kinase promotes microtubule outgrowth from chromosomes during spindle assembly. Chromosoma 2010; 119:415424.

21 Giet R, McLean D, Descamps S, et al. Drosophila Aurora A kinase is required to localize D-TACC to centrosomes and to regulate astral microtubules. J Cell Biol 2002; 156:437-451.

22 Barros TP, Kinoshita K, Hyman AA, Raff JW. Aurora A activates D-TACC-Msps complexes exclusively at centrosomes to stabilize centrosomal microtubules. J Cell Biol 2005; 170:1039-1046.

23 Kinoshita K, Noetzel TL, Pelletier L, et al. Aurora A phosphorylation of TACC $3 /$ maskin is required for centrosomedependent microtubule assembly in mitosis. J Cell Biol 2005; 170:1047-1055.

24 Pascreau G, Delcros JG, Cremet JY, Prigent C, Arlot-Bonnemains Y. Phosphorylation of maskin by Aurora-A participates in the control of sequential protein synthesis during Xenopus laevis oocyte maturation. J Biol Chem 2005; 280:1341513423.

25 LeRoy PJ, Hunter JJ, Hoar KM, et al. Localization of human TACC 3 to mitotic spindles is mediated by phosphorylation on Ser558 by Aurora A: a novel pharmacodynamic method for measuring Aurora A activity. Cancer Res 2007; 67:5362-5370.

26 Gergely F, Draviam VM, Raff JW. The ch-TOG/XMAP215 protein is essential for spindle pole organization in human somatic cells. Genes Dev 2003; 17:336-341.

27 Peset I, Seiler J, Sardon T, et al. Function and regulation of Maskin, a TACC family protein, in microtubule growth during mitosis. J Cell Biol 2005; 170:1057-1066.

28 Royle SJ, Lagnado L. Trimerisation is important for the function of clathrin at the mitotic spindle. J Cell Sci 2006; 119:4071-4078.

29 Schneider L, Essmann F, Kletke A, et al. The transforming acidic coiled coil 3 protein is essential for spindle-dependent chromosome alignment and mitotic survival. J Biol Chem 2007; 282:29273-29283.

30 Musacchio A, Salmon ED. The spindle-assembly checkpoint in space and time. Nat Rev Mol Cell Biol 2007; 8:379-393.

31 Nezi L, Musacchio A. Sister chromatid tension and the spindle assembly checkpoint. Curr Opin Cell Biol 2009; 21:785-795.

32 Medendorp K, Vreede L, van Groningen JJ, et al. The mitotic arrest deficient protein MAD2B interacts with the clathrin light chain A during mitosis. PLoS One 2010; 5:e15128.

33 Clarke PR, Zhang C. Spatial and temporal coordination of mitosis by Ran GTPase. Nat Rev Mol Cell Biol 2008; 9:464-477.

34 Santolini E, Puri C, Salcini AE, et al. Numb is an endocytic protein. J Cell Biol 2000; 151:1345-1352.

35 Sorensen EB, Conner SD. AAK1 regulates Numb function at an early step in clathrin-mediated endocytosis. Traffic 2008; 9:1791-1800.

36 Lee CY, Andersen RO, Cabernard C, et al. Drosophila AuroraA kinase inhibits neuroblast self-renewal by regulating aPKC/ Numb cortical polarity and spindle orientation. Genes Dev 2006; 20:3464-3474.

37 Wang H, Ouyang Y, Somers WG, Chia W, Lu B. Polo inhibits progenitor self-renewal and regulates Numb asymmetry by phosphorylating Pon. Nature 2007; 449:96-100.

38 Wirtz-Peitz F, Nishimura T, Knoblich JA. Linking cell cycle to asymmetric division: Aurora-A phosphorylates the Par complex to regulate Numb localization. Cell 2008; 135:161-173.

39 Royle SJ. The cellular functions of clathrin. Cell Mol Life Sci 2006; 63:1823-1832.

40 Mills IG. The interplay between clathrin-coated vesicles and cell signalling. Semin Cell Dev Biol 2007; 18:459-470.

41 Warner AK, Keen JH, Wang YL. Dynamics of membrane clathrin-coated structures during cytokinesis. Traffic 2006; 7:205-215.

42 Boucrot E, Kirchhausen T. Endosomal recycling controls plasma membrane area during mitosis. Proc Natl Acad Sci USA 2007; 104:7939-7944.

43 Cayrol C, Cougoule C, Wright M. The beta2-adaptin clathrin adaptor interacts with the mitotic checkpoint kinase BubR1. Biochem Biophys Res Commun 2002; 298:720-730.

44 Ford MG, Mills IG, Peter BJ, et al. Curvature of clathrincoated pits driven by epsin. Nature 2002; 419:361-366.

45 Jakobsson J, Gad H, Andersson F, et al. Role of epsin 1 in 
synaptic vesicle endocytosis. Proc Natl Acad Sci USA 2008; 105:6445-6450.

46 Liu Z, Zheng Y. A requirement for epsin in mitotic membrane and spindle organization. $J$ Cell Biol 2009; 186:473-480.

47 Tsai MY, Wang S, Heidinger JM, et al. A mitotic lamin B matrix induced by RanGTP required for spindle assembly. Science 2006; 311:1887-1893.

48 Ma L, Tsai MY, Wang S, et al. Requirement for Nudel and dynein for assembly of the lamin B spindle matrix. Nat Cell Biol 2009; 11:247-256.
49 Daub H, Olsen JV, Bairlein M, et al. Kinase-selective enrichment enables quantitative phosphoproteomics of the kinome across the cell cycle. Mol Cell 2008; 31:438-448.

50 Dephoure N, Zhou C, Villen J, et al. A quantitative atlas of mitotic phosphorylation. Proc Natl Acad Sci USA 2008; 105:10762-10767.

51 Seimiya M, Tomonaga T, Matsushita K, et al. Identification of novel immunohistochemical tumor markers for primary hepatocellular carcinoma; clathrin heavy chain and formiminotransferase cyclodeaminase. Hepatology 2008; 48:519-530. 